\title{
On features of crystal structure of semiconductor-ferroelectric $\mathrm{Ag}_{3} \mathrm{AsS}_{3}$
}

\author{
N. Borovoy ${ }^{1}$, Yu. Gololobov ${ }^{2}$, G. Isaienko² ${ }^{2}$ A. Salnik ${ }^{1 *}$ \\ ${ }^{1}$ Taras Shevchenko Kyiv National University, Faculty of Physics, \\ 4, prosp. Glushkova, Kyiv, Ukraine; *e-mail: alina.salnik@gmail.com \\ ${ }^{2}$ National Transport University, Faculty of Transport and Information Technologies, \\ 1, Suvorova str., 01011 Kyiv, Ukraine
}

\begin{abstract}
The temperature dependences of the unit cell parameters $a(T)$ and $c(T)$ of $\mathrm{Ag}_{3} \mathrm{AsS}_{3}$ were measured by the X-ray dilatometry method with high precision within the temperature range 100 to $300 \mathrm{~K}$ in the dark mode and under laser irradiation $(\lambda=$ $532 \mathrm{~nm})$. It was found that the parameter $c$ increases almost linear with decreasing the temperature from 300 down to $100 \mathrm{~K}$ for samples in the dark. At the same time, for samples that were exposed during cooling to laser irradiation, the increase of the parameter $c$ by the value $\Delta c \approx(0.002 \ldots 0.003) \AA$ is observed at temperatures $T_{p}=$ $145 \ldots 147 \mathrm{~K}$. This leap is typical for systems in which a phase transition of the first order occurs. Furthermore, there were investigated temperature dependences of the integral relative intensity $I(T)$ of main structural maxima of $\mathrm{Ag}_{3} \mathrm{AsS}_{3}$ both in the dark mode and under laser irradiation. It was established the character of the dependence $I(T)$ for these reflexes was changed significantly by laser irradiation.
\end{abstract}

Keywords: proustite, unit cell parameters, phase transition, photoinduced effects, integral intensity.

Manuscript received 09.07.13; revised version received 06.09.13; accepted for publication 19.09.13; published online 30.09.13.

\section{Introduction}

The crystals of proustite $\mathrm{Ag}_{3} \mathrm{AsS}_{3}$ are semiconductorsferroelectrics with high ionic conductivity, which are characterized by large optical anisotropy, pleochroism and transparency in infrared region. Because of these properties, proustite belongs to promising materials of nonlinear optics. That is why its physical properties are actively investigated within the temperature range 4.2 to $600 \mathrm{~K}[1,2]$. The crystals of proustite belong to the noncentro-symmetrical class of trigonal symmetry at room temperature (space group $C_{3 v}^{6}$ ) [2-4]. Temperature decrease of proustite is accompanied by structural phase transformations (PT), according to [3] at temperatures of $56 \mathrm{~K}$ (symmetry changing $\left.C_{3 v} \rightarrow C_{s}\right)$ and $24 \mathrm{~K}\left(C_{s} \rightarrow C_{1}\right)$.
Full-scale study of the heat capacity and the character of temperature transformation of NQR spectrum allowed authors [5] to firstly conclude about the existence of II order PT at $T_{i}=(60.5 \pm 0.5) \mathrm{K}$ and I order PT at $T_{N}=(49.5 \pm 0.5) \mathrm{K}$ in crystals of proustite and about the existence of an incommensurate phase between these PT. After that, formation of modulated structures were directly confirmed by X-ray diffraction and neutronographic methods at $T_{i}=60 \mathrm{~K}[6,7]$. Further investigations showed that the modulated structure is also incommensurate in the interval $T=28 \ldots 50 \mathrm{~K}$, but with rather a small period and orientation changes [8]. So, there is some sequence of PTs at temperatures $T<$ $100 \mathrm{~K}$ in crystals $\mathrm{Ag}_{3} \mathrm{AsS}_{3}$ : symmetric phase $\leftarrow 60 \mathrm{~K} \rightarrow$ incommensurate phase $\leftarrow 50 \mathrm{~K} \rightarrow$ new incommensurate 
phase $\leftarrow 28 \mathrm{~K} \rightarrow$ ferroelectric phase without structural modulation. There are different viewpoints about PT at temperatures $T>100 \mathrm{~K}$ in proustite in the literature.

The important feature of proustite is its crystal structure sensitivity to optical irradiation. Particularly, authors [3] first suggested existence of photoinduced changes at the temperature $T \approx 150 \mathrm{~K}$ in proustite crystals $\mathrm{Ag}_{3} \mathrm{AsS}_{3}$ based on revealed splitting of the Emode in the Raman spectra. The results of Raman scattering and Brillouin scattering investigations enabled to conclude about existence of the photoinduced PT in the temperature range $T=200 \ldots 210 \mathrm{~K}$ and propose another scheme of structural transformations: $C_{3 v} \rightarrow C_{s}$ (PT of the II order, $\left.T_{0}=210 \mathrm{~K}\right), C_{s} \rightarrow C_{s}$ (a cell doubling, PT of the II order, $T_{1}=56 \mathrm{~K}$ ), $C_{s} \rightarrow C_{1}$ (PT of the I order, $\left.T_{2}=24 \mathrm{~K}\right)[4,9,10]$. But, the anomalies that are typical for PT, were observed in the temperature dependences of the velocity of longitudinal ultrasonic waves in irradiated proustite samples at the temperature $T \approx 150 \mathrm{~K}[11]$.

So, there is no single viewpoint about character and mechanisms of temperature and optical irradiation effect on physical properties of proustite crystals in the literature. Furthermore, the precision measurements of the unit cell parameter $a$ and $c$ of proutite crystals at temperature $T<300 \mathrm{~K}$ were not represented in the literature at all. Therefore, in this work the temperature dependences of the unit cell parameters $a(T)$ and $c(T)$ were investigated using the X-ray dilatometry method, also determined were integral relative intensities of some main structural maxima of proustite crystals within the temperature range $100<T<300 \mathrm{~K}$ both in the dark mode and under laser irradiation.

\section{Experimental}

The investigated samples were grown by the BridgmanStockbarger method. The X-ray diffraction patterns of $\mathrm{Ag}_{3} \mathrm{AsS}_{3}$ were obtained using DRON-4-07 diffractometer ( $\mathrm{Fe} \mathrm{K} \alpha_{1,2}$ radiation). To study the dependences $a(T)$ and $c(T)$, single crystals of proustite were used, in which planes $(00 l)$ and $(h 00)$ were derived on the physical surface. To obtain the dependence $c(T)$, the reflection (006) was registered, and in case $a(T)-$ reflection (10.00).

Reflections were scanned in steps $\delta(2 \theta)=0.02^{\circ}$, the accumulation of impulses at a point was $2 \mathrm{~s}$. The functions of Voigt, which fitted the compounds $K \alpha_{1}$ and $K \alpha_{2}$, were used during separation of $K \alpha_{1,2}$-doublet. The accuracy in determining the lattice parameters $a$ and $c$ was not worse than $0.002 \AA$. The study of the temperature dependences of the relative integral intensity $I(T)$ of structural maxima were performed on polycrystalline samples that were obtained by grinding single crystals $\mathrm{Ag}_{3} \mathrm{AsS}_{3}$. The diffraction patterns of polycrystals were obtained in some angular intervals of the width $\Delta(2 \theta)=(3 \ldots 5)^{\circ}$, which corresponded to the structural maxima (122), (232), (312) and (202) in steps $\delta(2 \theta)=0.03^{\circ}$, the accumulation time at the point was $10 \ldots 30 \mathrm{~s}$. Samples were placed in a URNT-180 standard low-temperature chamber. The sample temperature was maintained with the accuracy $0.5 \mathrm{~K}$ using a VRT-2 thermoregulator and measured by copper-constantan thermocouple. Optical irradiation of samples during cooling and heating was carried out using the MGL 500 milliwatt laser $(\lambda=532 \mathrm{~nm})$, irradiation power incident on the sample was $40 \mathrm{~mW}$.

\section{Results and discussions}

Fig. 1 shows temperature dependences of the unit cell parameter $a(T)$, which were obtained during samples cooling: (a) - dark mode; (b) - under laser irradiation.

As can be seen, dependences $a(T)$ are close to linear, and the value of parameter $a$ is almost the same in the dark mode and under laser irradiation. At the same time, there was observed a weak change in the slope of curve $a(T)$ at the temperatures $T=170 \ldots 200 \mathrm{~K}$ on both figures, so the slope angle is smaller at $T>200 \mathrm{~K}$ than $T<170 \mathrm{~K}$. It should be noted that dependences obtained during heating almost do not differ from the above.
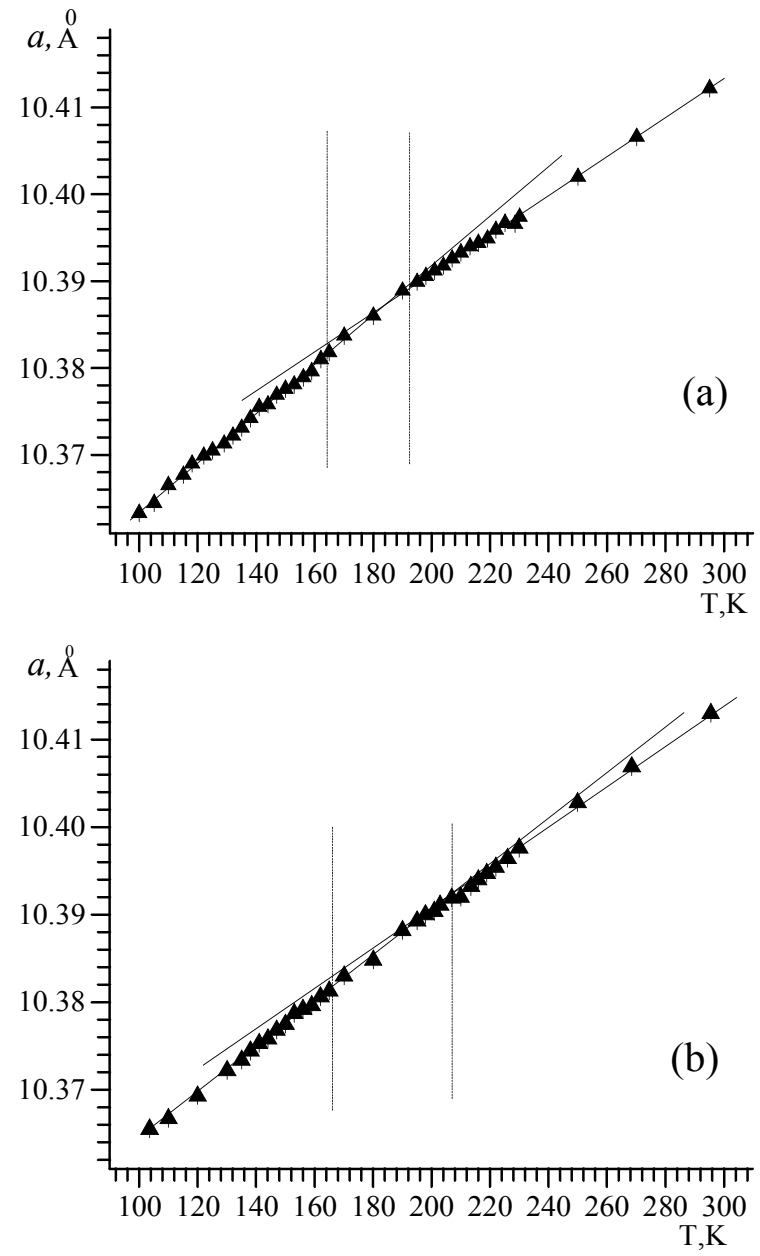

Fig. 1. Temperature dependences $a(T)$ for $\mathrm{Ag}_{3} \mathrm{AsS}_{3}$ : a) dark mode; b) under laser irradiation. 
Temperature dependence of the unit cell parameter $c(T)$ of proustite crystals both in the dark mode and under laser irradiation of the sample (cooling mode) is shown in Fig. $2 a$ and $2 b$, respectively. First of all, there are two temperature ranges with significantly different slope angles of $c(T)$ : interval $\Delta T_{1}=220 \ldots 300 \mathrm{~K}$, where the parameter $c$ in general slightly varies with temperature, and interval $\Delta T_{2}=100 \ldots 170 \mathrm{~K}$, where the slope angle is significant. The slope angle of the dependence $c(T)$ is monotonically changed within the temperature range 170 to $220 \mathrm{~K}$. Thus, the coefficient of thermal expansion $\alpha_{c}=\partial c / \partial T$ of $\mathrm{Ag}_{3} \mathrm{AsS}_{3}$ crystals along the axis $C$ was significantly different in two temperature ranges $\Delta T_{1}$ and $\Delta T_{2}$ : $\alpha_{c}\left(\Delta T_{1}\right)=3.5 \cdot 10^{-5} \AA / \mathrm{K} ; \alpha_{c}\left(\Delta T_{2}\right)=1.4 \cdot 10^{-4} \AA / \mathrm{K}$.

It should be noted that in the paper [1], the parameters $a$ and $c$ were determined with poor experimental accuracy, so the changing of angle slope $c(T)$ was not observed within the temperature range 170 to $220 \mathrm{~K}$. It is important that the character of the dependence $c(T)$ is practically the same for both samples in the dark mode and under laser irradiation.

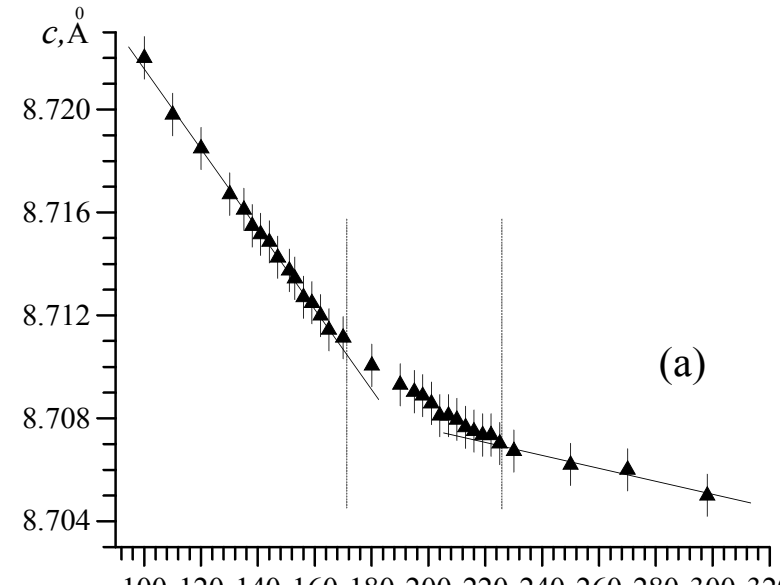

100120140160180200220240260280300320

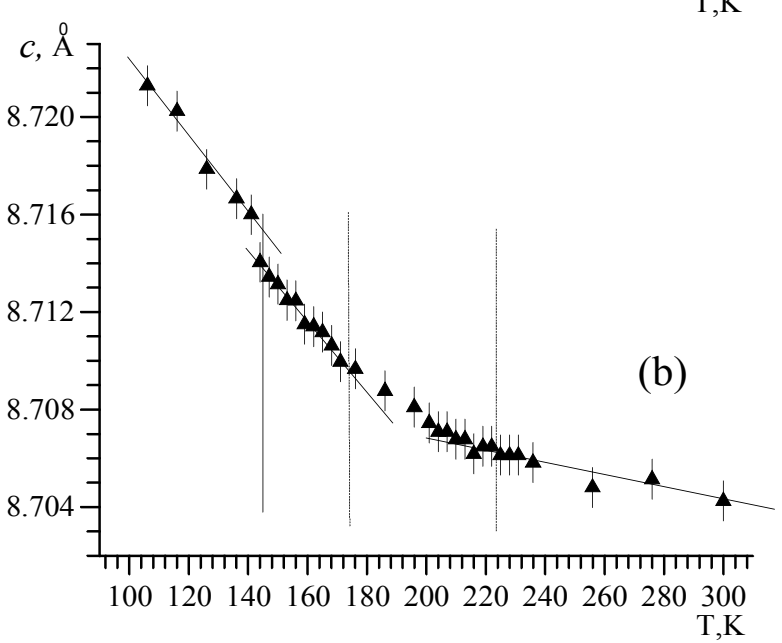

Fig. 2. Temperature dependences $c(T)$ for $\mathrm{Ag}_{3} \mathrm{AsS}_{3}$ : a) dark mode; b) under laser irradiation.

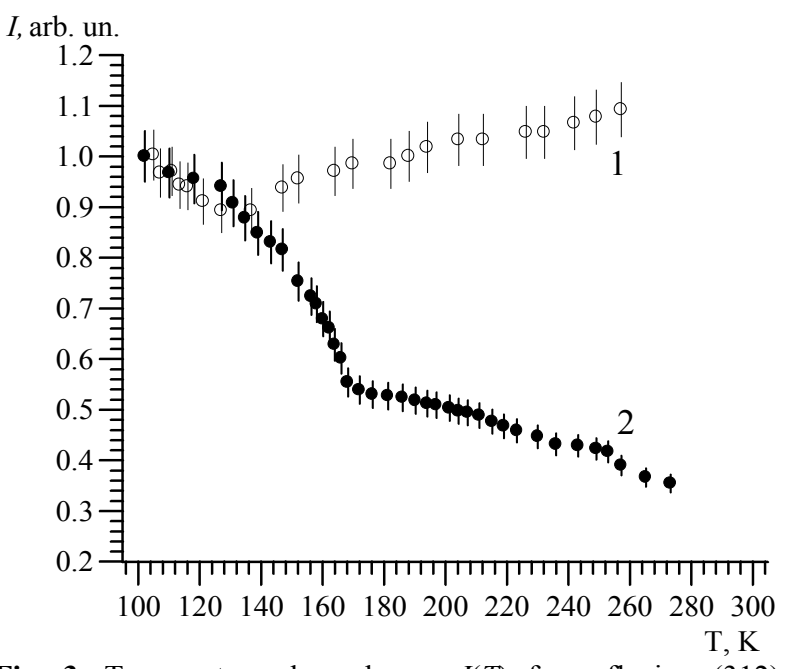

Fig. 3. Temperature dependences $I(T)$ for reflexion (312): 1) dark mode; 2) under laser irradiation.

However, there observed was an important difference between them for temperatures $T<170 \mathrm{~K}$. Namely, parameter $c$ monotonically increases with temperature decreasing for samples within the temperature range 100 to $170 \mathrm{~K}$ in the dark. At the same time, there is an abrupt increase of the parameter $\Delta c \approx$ $0.002 \ldots 0.003 \AA$ at temperatures 144 to $146 \mathrm{~K}$ for samples that were laser irradiated during the cooling process. This leap of unit cell parameters values is typical for systems in which phase transitions of the first order occur.

We have previously established that dependences $I(T)$ for reflections (122), (232), (312) and (202) are fundamentally different for polycrystalline samples of proustite in the dark mode in the course of cooling in two temperature regions: integral intensities for all four maxima decrease from room temperature down to $140 \ldots 160 \mathrm{~K}$ (an abnormal dependences), and with further cooling down to $100 \mathrm{~K}$ integral intensities grow [12]. In this work, the temperature dependences $I(T)$ were measured for mentioned maxima both in the dark mode and under laser irradiation. It was revealed that the character of the dependence $I(T)$ significantly change: integral intensities for all maxima increase within the temperature range 300 to $100 \mathrm{~K}$ during the cooling process. In Fig. 3 this effect is illustrated for the reflection (312).

The nature of revealed photoinduced effects - like leap in the dependences $c(T)$ at the temperature $T=$ $144 \ldots 146 \mathrm{~K}$ and differences in the dependence $I(T)$, may be related to the process of photostimulated migration of $\mathrm{Ag}^{+}$ions on crystallographic positions in the unit cell (the depth of the potential well for $\mathrm{Ag}^{+}$in the unit cell is less than $\Delta U=0.7 \ldots 1.0 \mathrm{eV}[1,2])$. The analysis of the structure amplitude in the dark mode of proustite showed that there are transitions of $\mathrm{Ag}^{+}$ions at temperatures $T \geq$ $150 \mathrm{~K}$ from main crystallographic to vacant positions, in general right-screwed spirals $(\mathrm{AgS})_{\infty}$. In particular, it 
causes abnormal behavior of dependences $I(T)$ for maxima (122), (232), (312) and (202). However, the simultaneous action of laser irradiation and temperature leads to disordering cationic sublattice of proutite at $T \geq$ $150 \mathrm{~K}$, i.e. accelerates the output of silver ions with equal probability from both right- and left-screwed spirals $(\mathrm{AgS})_{\infty}$. In this case, $\mathrm{Ag}^{+}$ions can move to vacant positions within the unit cell, or go in a structure heterogeneity area and on the surface of crystallites.

This disordering the cationic sublattice should be accompanied by decrease in the integral intensity of these reflections with temperature increasing, as observed in this work. The temperature match of the leap in the dependence $c(T)$ and inflection of the dependence $I(T)(T \approx 145 \ldots 150 \mathrm{~K})$ is an additional evidence that the character of $\mathrm{Ag}^{+}$ions migration qualitatively changes between right- and left-screwed spirals $(\mathrm{AgS})_{\infty}$ exactly in this temperature range.

It should also be noted that contradictory data known in the literature about the existence of photoinduced changes in physical parameters of proustite within the temperature interval 200 to $210 \mathrm{~K}$ $[1,2]$ may be caused by the "transitional" state of proustite crystals within the temperature range 170 to $220 \mathrm{~K}$. Indeed, the coefficient of thermal expansion of proutite $\alpha_{c}$ varies more than three times within the mentioned temperature range, which is typical for the blurred phase transition. Therefore, optical irradiation of proustite crystals in this state, depending on parameters of a defect subsystem, may cause appearance of various anomalies in temperature dependences of electrical, thermal and optical properties.

\section{Conclusions}

Photoiduced leaped increasing of the unit cell parameter $c$ was experimentally observed at the temperature $T=$ (144...146 K for the first time, which confirmed the existence of photoinduced phase transition of the first order in proustite crystals. It was experimentally revealed the existence of two temperature ranges $220 \ldots 300 \mathrm{~K}$ and $100 \ldots 170 \mathrm{~K}$, in which the value of the thermal expansion coefficient for proustite crystals changed thrice. The change in temperature dependences of integral intensities was revealed under laser irradiation for several structural maxima of proustite crystals, which are caused by the action of optical irradiation on processes of thermal migration of silver ions in the proustite unit cell.

\section{References}

1. K.A. Schonau and S.A.T. Derfern, Hightemperature phase transitions, dielectric relaxation, and ionic mobility of proustite, $\mathrm{Ag}_{3} \mathrm{AsS}_{3}$, and pyrargyrite, $\mathrm{Ag}_{3} \mathrm{SbS}_{3} / / \mathrm{J}$. Appl. Phys. 92(12), p. 7415-7424 (2002).

2. A. Gagor, A. Pawowski and A. Pietraszko, Silver transfer in proustite $\mathrm{Ag}_{3} \mathrm{AsS}_{3}$ at high temperatures: Conductivity and single-crystal X-ray studies // J. Solid State Chem. 182, p. 451-456 (2009).

3. G.A. Smolenskiy, I.G. Siniy, E.G. Kuzminov and A.A. Godovikov, Optical phonons and soft mode in proustite at phase transition // Phys. Solid State 21(8), p. 2338-2341 (1979).

4. G.A. Smolenskiy, I.G. Siniy, S.D. Prohorova, E.G. Kuzminov and A.V. Godovikov, New phase transition in proustite // Crystalographia 27(1), p. 140-145 (1982), in Russian.

5. A.B. Bondar, V.S. Vihrin, S.M. Riabchenko and V.E. Iachmenev, Incommensurate phase near phase transition of the second order in proustite // Phys. Solid State 25(9), p. 2602-2609 (1983).

6. S.S. Hasanov, V.Sh. Shlehtman and I.M. Shmytko, Creation of modulated structure in proustite // Phys. Solid State 26(3), p. 935-938 (1984).

7. R.J. Nelmest, C.J. Howard, T.W. Ryan, W.I.F. David, A.J. Schultz and P.C.W. Leung, A neutron and diffraction study of the phase transitions in proustite $\left(\mathrm{Ag}_{3} \mathrm{AsS}_{3}\right)$ between $35 \mathrm{~K}$ and room temperature // J. Phys. C: Solid State Phys. 26(3), p. 861-865 (1984).

8. I.M. Shmytko, V.Sh. Shlehtman, B.Sh. Bagautdinov and N.S. Afonikova, Dinamic hysteresis effects near existence of modulated structure in proustite // Phys. Solid State 32(8), p. 2441-2447 (1990).

9. L.A. Kot, S.D. Prohorova, Iu.M. Sandler, I.G. Siniy and I.N. Flerov, Photostimulated phase transition in proustite // Phys. Solid State 28(5), p. 1535-1537 (1983).

10. Ia. Shauren and K.N. Teylor, Studing og photostimulated phase transition in proustite $\mathrm{Ag}_{3} \mathrm{AsS}_{3} / /$ Phys. Solid State 28(9), p. 2604-2607 (1986).

11. Iu.P. Gololobov, On photostimulated phase transition in $\mathrm{Ag}_{3} \mathrm{AsS}_{3}$ crystal // Phys. Solid State 41(4), p. 702-704 (1999).

7. N.A. Borovoy, Iu.P. Gololobov and I.N. Salivonov, Termorearrangement of cation sublattice in proustite // Low Temp. Phys. 25, p. 546-549 (1999). 